\title{
ON A CONJECTURE OF MELZAK
}

\author{
G. C. Shephard \\ (received March 7, 1964)
}

Melzak [2] has shown that there exists a convex pseudopolyhedron $Q$ (the convex hull of a convergent sequence of points together with its limit point) in $E_{3}$ which is s-universal for triangles, that is, all possible triangles occur (up to similarity) as plane sections of $Q$. He conjectured that no polyhedron $P$ has this property. In this short note we give an elementary proof of this conjecture. (In [1], Klee discusses in detail the problem of the existence of s-universal polyhedra for various classes $K$ of convex bodies. He asserts on p. 263, but does not prove, that Melzak's conjecture is true.)

The proof is by contradiction. Suppose a bounded convex polyhedron $P$ (the convex hull of a finite number of points) in $E_{3}$ is s-universal for triangles. Let $\left\{\alpha_{i}\right\}$ be any monotonic decreasing sequence of real numbers, each less than $\frac{1}{2} \pi$, tending to the limit 0 as $i \rightarrow \infty$. Let $\Delta_{i}$ be an isosceles triangle with angles $\alpha_{i}, \alpha_{i}, \pi-2 \alpha_{i}$, and let $\delta_{i}$ be the plane section of $P$ similar to $\Delta_{i}$. We shall refer to the vertex of $\delta_{i}$ where the angle $\pi-2 \alpha_{i}$ occurs as the apex of the triangle, the opposite side as the base of the triangle, and the distance between these two as the height of the triangle. Since the sides of $\delta_{i}$ lie on the faces of $P$ there is a correspondence

$$
\Delta_{i} \leftrightarrow\left(p_{1}^{i}, p_{2}^{i} ; q^{i}\right)
$$

between the triangles $\Delta_{i}$ and the semi-ordered triples of faces

Canad. Math. Bull. vol. 7, no.4, October 1964 
of $P$ (semi-ordered because $\Delta_{i}$ is isosceles so the order of $p_{1}^{i}, p_{2}^{i}$ is not significant). As $P$ has a finite number of faces there are only a finite number of the se semi-ordered triples, and so some subsequence of $\left\{\Delta_{i}\right\}$ consists of triangles cor. responding to the same triple $\left(p_{1}, p_{2} ; q\right)$. By change of notation we may write $\left\{\Delta_{i}\right\}$ for this subsequence.

Two cases now arise:

1. The plane $q$ is parallel to the line $\ell=p_{1} \cap p_{2}$. Let $d$ be the distance between $q$ and $\ell$; then it is easy to see that any plane section of $p_{1}, p_{2}, q$ must be a triangle whose height is at least $d$. This means that $\delta_{i}$ has height at least $d$ and base at least $2 \mathrm{~d} \cot \alpha_{i}$. As $i \rightarrow \infty, \alpha_{i} \rightarrow 0,2 \mathrm{~d} \cot \alpha_{i} \rightarrow \infty$ and so the sequence of triangles $\left\{\delta_{i}\right\}$ is unbounded. This is a contradiction since we have assumed that $P$ is bounded.

2. The plane $\mathrm{q}$ is not parallel to the line $\ell$. Let a variable plane $h$ cut the faces $p_{1}, p_{2}$ in lines $m_{1}, m_{2}$ making angles $\varphi_{1}, \varphi_{2}$ with $\ell$ respectively (see the diagram). If $h$ varies in such a manner that $\hat{m_{1} m_{2}} \rightarrow \pi$, then it is easy to see that either

$$
\varphi_{1} \rightarrow 0 \text { and } \varphi_{2} \rightarrow \pi \text { or } \varphi_{1} \rightarrow \pi \text { and } \varphi_{2} \rightarrow 0
$$

But if $h$ meets $q$ neither of these possibilities can occur, since if $\theta_{1}, \theta_{2}$ are the angles between $q \cap p_{1}, q \cap p_{2}$ and $l$

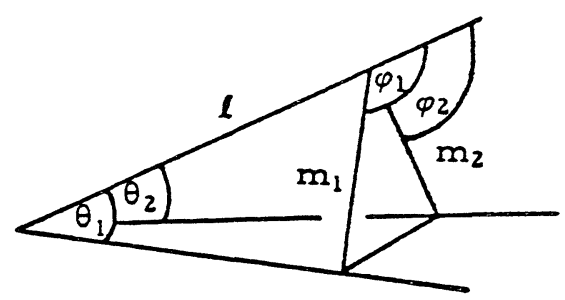


respectively, then $\varphi_{1}>\theta_{1}$ and $\varphi_{2}>\theta_{2}$. We conclude that $\widehat{m_{1} m_{2}}$ is bounded away from $\pi$. This is a contradiction since, by our original assumption, the triangles $\delta_{i}$ (whose apex angles are arbitrarily close to $\pi$ ) occur amongst these sections.

Hence each of the two cases leads to a contradiction and the conjecture is proved.

The boundedness of $P$ is an essential condition for the truth of the conjecture. A simple continuity argument establishes that any infinite triangular prism is $s$-universal for triangles. Further, if $P$ is a tetrahedron at one vertex of which the three face angles are $\alpha(0<\alpha<\pi / 3)$, then $P$ is $s$-universal for triangles whose angles all exceed $\alpha$.

\section{REFERENCES}

1. V. Klee, Polyhedral sections of convex bodies. Acta Math. 103 (1960), 243-267.

2. Z.A. Melzak, A property of convex pseudopolyhedra. Canadian Bull. Math. 2 (1959), 31-32.

University of Birmingham 